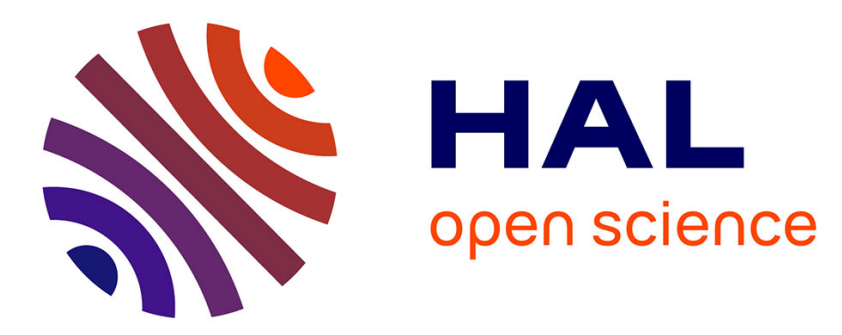

\title{
Note: Localization based on estimated source energy homogeneity
}

Semih Turkaya, Renaud Toussaint, Fredrik Kvalheim Eriksen, Olivier Lengliné, Guillaume Daniel, Eirik G. Flekkøy, Knut Jørgen Måløy

\section{- To cite this version:}

Semih Turkaya, Renaud Toussaint, Fredrik Kvalheim Eriksen, Olivier Lengliné, Guillaume Daniel, et al. Note: Localization based on estimated source energy homogeneity. Review of Scientific Instruments, 2016, 87 (9), pp.5563 - 5571. 10.1063/1.4962407 . hal-01628817

\section{HAL Id: hal-01628817 https://hal.science/hal-01628817}

Submitted on 4 Nov 2017

HAL is a multi-disciplinary open access archive for the deposit and dissemination of scientific research documents, whether they are published or not. The documents may come from teaching and research institutions in France or abroad, or from public or private research centers.
L'archive ouverte pluridisciplinaire HAL, est destinée au dépôt et à la diffusion de documents scientifiques de niveau recherche, publiés ou non, émanant des établissements d'enseignement et de recherche français ou étrangers, des laboratoires publics ou privés. 


\section{NOTE: Localization Based On Estimated Source Energy Homogeneity}

Semih Turkaya ${ }^{1, a)}$ and Renaud Toussaint ${ }^{1}$, Fredrik Kvalheim Eriksen ${ }^{1,2}$, Olivier Lengliné ${ }^{1}$, Guillaume Daniel ${ }^{3}$, Eirik G. Flekk $\varnothing \mathrm{y}^{2}$, Knut Jørgen Målø ${ }^{2}$

${ }^{1} I P G$ Strasbourg, CNRS, Université de Strasbourg, Strasbourg, France

${ }^{2}$ Department of Physics, University of Oslo, Oslo, Norway

${ }^{3}$ Magnitude, Sainte Tulle, France

(Dated: May 2016)

Acoustic signal localization is a complex problem with a wide range of industrial and academic applications. Herein, we propose a localization method based on energy attenuation and inverted source amplitude comparison (termed estimated source energy homogeneity, or ESEH). This inversion is tested on both synthetic (numerical) data using a Lamb wave propagation model, and experimental 2D plate data (recorded with 4 accelerometers sensitive up to $26 \mathrm{kHz}$ ). We compare the performance of this technique with classic source localization algorithms: arrival time localization, time reversal localization, and localization based on energy amplitude. Our technique is highly versatile, and out-performs the conventional techniques in terms of error minimization and cost (both computational and financial).

Source localization of acoustic signals is fundamental for a broad range of academic and industrial applications, from robotics to medicine; from telecommunications to geoscience $^{1-7}$. Even though it is trivial to locate impulsive, non-dispersive signals without any reflections ${ }^{8}$, as the signal and propagating medium get more complex this task becomes more difficult. In this paper, a new approach of localization based on the signal energy is presented. It is possible to implement this estimated source energy homogeneity (ESEH) method into the authors' previous work ${ }^{9}$ to localize microseismic events in aerofracturing of a porous medium in a Hele-Shaw Cell ${ }^{10}$, or other types of $2 \mathrm{D}$ experiments such as fracture propagation experiments in plexiglass ${ }^{11}$ or crack propagation in paper $^{12}$. Furthermore, it is possible to implement this method into a 3D medium at a larger scale which then can be used in the industrial applications such as geothermal activities ${ }^{13-15}$, well stimulation ${ }^{16}$ etc.

We here present a set of experimental and numerical results that compare different methods of localization: Arrival Time Delay Localization (ATL) ${ }^{17-22}$, Energy Based Localization (EBL) ${ }^{23,24}$, Time Reversal Localization (TRL) ${ }^{6,7,25}$ (see Supplementary Material S1), and ESEH.

A glass (or plexiglass) plate having dimensions $80 \mathrm{~cm} \times$ $40 \mathrm{~cm} \times 1 \mathrm{~cm}$ is used for experiments. A $4.5 \mathrm{~mm}$ diameter steel ball is dropped from $2-3 \mathrm{~cm}$ height above the plate on different locations. The signal generated by the impact is recorded with a set of 4 miniature piezoelectric accelerometers (Brüel \& Kjaer - 4374) with an optimal

a)Electronic mail: turkaya@unistra.fr sensitivity in the $(1 \mathrm{~Hz}-26 \mathrm{kHz})$ frequency range. The recorded signal is amplified and conditioned using a Brüel \& Kjaer Nexus Charge Amplifier - Type 2692-A. Then, the signals are transmitted to the computer using a NiDAQ mx PCI-6133 acquisition card.

This new method relies on the principle that the source energy, after correction from travel path-related attenuation, should be equal (or almost equal) at every sensor location. The energy spreading due to distance for plates can be defined as $E_{m}=\frac{E}{2 \pi R h}$ where $E_{m}$ is the source energy $E$ spread to a receiver $m$ at a distant $R$ where $h$ is the plate thickness. In this new approach, the energy is calculated using the equation ${ }^{26,27}$ :

$$
E_{s}\left(\mathbf{r}_{\mathbf{s}}, \mathbf{r}_{\mathbf{n}}\right)=\int_{0}^{\omega_{N y q}} 2 \pi R(n) \rho h c(\omega) \frac{|a(\omega)|^{2}}{\omega^{2}} e^{\alpha(\omega) R(n)} d \omega
$$

where $E_{s}\left(\mathbf{r}_{\mathbf{s}}, \mathbf{r}_{\mathbf{n}}\right)$ is the energy of the source seen from the sensor number $\mathrm{n}$ at position $\mathbf{r}_{\mathbf{n}}$, if the source is at position $\mathbf{r}_{\mathbf{s}}, \rho$ is the mass density of the plate, $c(\omega)$ is the group velocity over different $\omega$ (angular frequency) at which the energy is traveling ${ }^{27}, a(\omega)$ is the acceleration of the plate after Fourier transform, $R(n)=\left\|\mathbf{r}_{\mathbf{n}}-\mathbf{r}_{\mathbf{s}}\right\|$ is the distance between the source and the receiver $n$ and $\omega_{N y q}$ is the Nyquist frequency $\left(\omega_{N y q}=\frac{\pi}{d t}\right.$ where $d t$ is a discrete time step). The part $e^{\alpha(\omega) R}$ represents correction due to the material based attenuation which is equal to 1 in the case of glass plate ${ }^{27}$. For plexiglass plate for the frequencies lower than the cutoff frequency $\omega_{c}=\omega(1 / h)$, this attenuation coefficient is equal to $\alpha(\omega)=1 \mathrm{~m}^{-1}$, and for the frequencies higher than cutoff frequency it is $\alpha(\omega)=7.64 \times 10^{-4} \omega^{2 / 3} \mathrm{~m}^{-127}$. Defining an average operator as $\langle A\rangle=\frac{1}{N} \sum_{n^{\prime}=1}^{N} A\left(n^{\prime}\right)$. and using the normalized source energy $\Psi_{s}\left(\mathbf{r}_{\mathbf{s}}, \mathbf{r}_{\mathbf{n}}\right)=$ $E_{s}\left(\mathbf{r}_{\mathbf{s}}, \mathbf{r}_{\mathbf{n}}\right) / \max \left(E_{s}\left(\mathbf{r}_{\mathbf{s}}, \mathbf{r}_{\mathbf{n}}\right)\right)$ where $\max \left(E_{s}\left(\mathbf{r}_{\mathbf{s}}, \mathbf{r}_{\mathbf{n}}\right)\right)$ is the maximum over the $N$ sensors, we can formulate the standard deviation of the energy recorded at $N$ different sensors as $\sigma\left(\mathbf{r}_{\mathbf{s}}\right)=\sqrt{\frac{1}{\mathbf{N}} \sum_{\mathbf{n}=\mathbf{1}}^{\mathbf{N}}\left(\boldsymbol{\Psi}_{\mathrm{s}}\left(\mathbf{r}_{\mathrm{s}}, \mathbf{r}_{\mathbf{n}}\right)-\left\langle\boldsymbol{\Psi}_{\mathrm{s}}\left(\mathbf{r}_{\mathrm{s}}, \mathbf{r}_{\mathbf{n}}\right)\right\rangle\right)^{\mathbf{2}}}$. The minimum of this standard deviation, $\sigma\left(\mathbf{r}_{\mathbf{s}}\right)$ over the plate will indicate the position of the source. This method is based on the direct wave (signal without the arrival of reflected waves from the sides). Therefore, it is necessary to carefully define the time window prior to the arrival of reflection so as to achieve high quality estimates of the source position. We define a direct wave window ranging from the impact duration of the source by using hertzian mechanics $(30 \mu s$ and $96 \mu s$ for the glass and plexiglass plates respectively $)^{28,29}$ up to the time of arrival of the reflected wave i.e. $\tau_{\text {source }}<L / V_{R}$, where $L$ is the length of the raypath of the closest reflected wave (97 $\mu s$ and $375 \mu s$ for the glass and plexiglass plates respec- 
tively). We used window sizes $50 \mu \mathrm{s}$ on glass and $200 \mu \mathrm{s}$ on plexiglass for the experimental cases. The Rayleigh wave velocity is $V_{R_{\text {glass }}} \approx 3100 \mathrm{~m} / \mathrm{s}$ and $V_{R_{\text {plexi }}} \approx 800$ $\mathrm{m} / \mathrm{s}$ on glass and plexiglass plates ${ }^{27}$.

Tests on synthetic data using hertzian contact load during shocks ${ }^{28,29}$ are conducted to check different methods (ATL, TRL, EBL, and ESEH) in different conditions before testing in physical experiments (see Supplementary Material S2). During the localization estimations, we used Rayleigh wave velocity $V_{R}$ as the wave velocity. However, it is possible to implement different group velocities for different frequencies in ESEH which decreases the error around $\approx 10 \mathrm{~mm}$. In table I, the average error of the different cases of signal generation are presented in this order: infinite and non-dispersive plate, infinite and dispersive plate by taking into account different velocities over the frequency spectrum, finite and non-dispersive plate by taking into account the reflected waves from the boundaries, and finite and dispersive plate by taking into account both. Signals for 18 various source locations are generated and localized using different methods. From

TABLE I. Table showing the average distance between the real source position and the estimated position (i.e. error) in $\mathrm{mm}$ for different cases in the signal generation.

\begin{tabular}{|l|l|l|l|l|}
\hline Mean Error, Glass Plate $(\mathrm{mm})$ & ATL & TRL & EBL & ESEH \\
\hline Infinite, Non-Dispersive & 0 & 0 & 7 & 0 \\
\hline Infinite, Dispersive & 45 & 26 & 35 & 4 \\
\hline Finite, Non-Dispersive & 2 & 2 & 20 & 5 \\
\hline Finite, Dispersive & 35 & 27 & 50 & 36 \\
\hline Experimental & 21 & 29 & 48 & 33 \\
\hline Mean Error, PlexiGlass Plate (mm) & ATL & TRL & EBL & ESEH \\
\hline Infinite, Non-Dispersive & 0 & 0 & 7 & 0 \\
\hline Infinite, Dispersive & 30 & 61 & 27 & 11 \\
\hline Finite, Non-Dispersive & 0 & 0 & 21 & 3 \\
\hline Finite, Dispersive & 36 & 37 & 48 & 40 \\
\hline Experimental & 48 & 34 & 34 & 30 \\
\hline
\end{tabular}

table I it can be seen that the effect of uncertainty due to the estimation of the source energy in the conventional method leads to some estimation error even in the simplest case. In the infinite cases where the reflections are not included, energy based methods are more precise. A clear definition of the primary signal means that ESEH performs well in all circumstances. ATL is comparable to ESEH when the medium is non-dispersive; however, with increased dispersivity the performance of ATL decreases due to the attendant uncertainty in the wave velocity.

In table II it is possible to compare computational costs for each method in every signal generation context:

This table is obtained by measuring the time spent during the estimation process with different methods. The window size of the signal is optimized for the cases to better include the direct wave. As a result, some of the less complex cases have longer signals and thus, take more time to estimate. From table II it can be seen that ATL is the cheapest method while EBL is the most expensive.

We tested experimentally the effect of the ball size $(1 \mathrm{~mm}, 5 \mathrm{~mm}$ and $10 \mathrm{~mm})$ and stiffness of the plate
TABLE II. Table showing the computational time in miliseconds for different cases.

\begin{tabular}{|l|l|l|l|l|}
\hline Mean Computational Cost (ms) & ATL & TRL & EBL & ESEH \\
\hline Infinite, Non-Dispersive & 6 & 31 & 5567 & 125 \\
\hline Infinite, Dispersive & 6 & 31 & 5567 & 125 \\
\hline Finite, Non-Dispersive & 6 & 43 & 6983 & 257 \\
\hline Finite, Dispersive & 6 & 22 & 4760 & 90 \\
\hline
\end{tabular}

(polyamide, glass and steel) of the signals. We observed that these properties do not affect localization results significantly. The average estimation error for different sampling rates for a set of experiments using a $5 \mathrm{~mm}$ steel ball hitting on a plexiglass plate are presented in Figure 1.

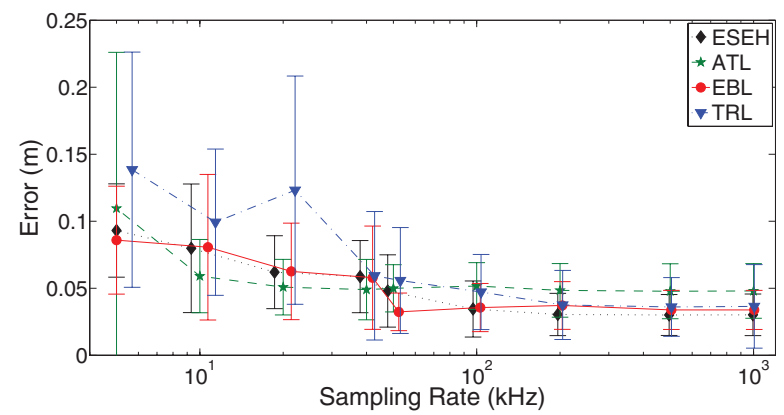

FIG. 1. Figure showing the decay of the average error on plexiglass plate with different sampling rates in different methods. Signals with lower sampling rates are obtained by decimating the experimentally recorded signal with $1 \mathrm{MHz}$ sampling rate.

On the other hand, due to the lower Rayleigh wave velocity $(\approx 800 \mathrm{~m} / \mathrm{s})$ in plexiglass, direct wave is clearer. This allows energy based methods to work in this case with a resolution $\approx 3.3 \pm 1.0 \mathrm{~cm}$, which is calculated by averaging the difference between the real and estimated source position for all the experiments (including the human error on targeting the predefined source position). Analysis of the experiments with different spatial resolution (by changing the grid from $1 \mathrm{~cm}$ to $1 \mathrm{~mm}$ ) has a minor effect on the resolution of the data, but it increases the computational cost 70 times (see Supplementary Material S3). The conventional energy based localization method can be optimized by having a linearly spaced grid of 10 points between the maximum and the minimum possible source energy (see Supplementary Material S4).

The sampling rate of the signal has a significant effect on the estimation process: the smaller the sampling rate, the higher the error on localization. However, EBL and ESEH are the most stable techniques and provide better results over the range of sampling rates tested.

To see the effect of signal to noise ratio in localization, Gaussian white noise with different amplitude are added to the input data. In figure 2 it can be seen that the average error from ATL is the largest when the signal quality is poor. Here, ESEH and EBL have very similar responses. Moreover, no dependence on the propagation medium (i.e. glass or plexiglass) has been observed for 
the decay of the localization error with respect to the signal to noise ratio (see Supplementary Material S5).

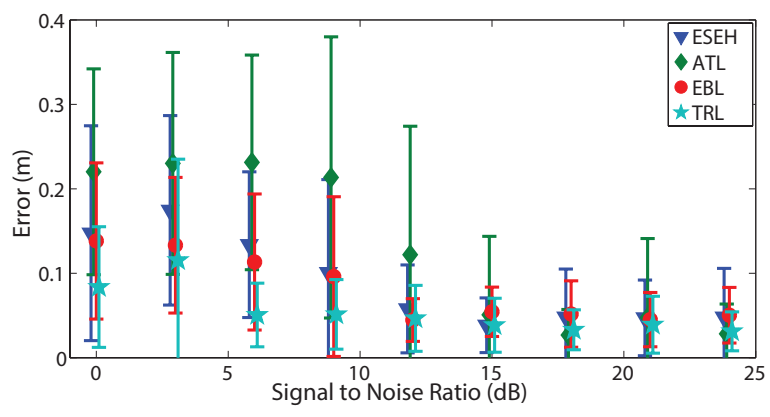

FIG. 2. Figure showing the effect of the signal to noise ratio (added artificially) of the signal over the estimation quality in different methods on glass plate.

To see the effect of array geometry on the resolution we placed the sensors in different positions over the plate in the simulations. Based on our investigations, we found out that a ' $\mathrm{T}$ ' shaped sensor placement gives the best results $(\approx 10 \%$ better estimation) (see Supplementary Material S6).

The theoretical formulation of ESEH can be used in 3D media by changing the cylindrical energy spreading (with a decay of the energy in $1 / R$ ) to spherical spreading (for deep sources: $E_{m}=\frac{E}{\frac{4}{3} \pi R^{2}}$ or for superficial sources: $\left.E_{m}=\frac{E}{\frac{2}{3} \pi R^{2}}\right)$ and changing the material based viscosity , i.e. attenuation (from 2D glass or plexiglass used in this research to the selected 3D medium). The two main advantages of employing a 3D medium are that (1) the signals reflected from the sides are-generally-clearly distinguishable (or not existing at all when boundaries are sufficiently distant), and (2) the waves are body waves, meaning that the medium is non-dispersive. We thus, anticipate improved resolution in a $3 \mathrm{D}$ medium compared to a system of plates.

The proposed method, ESEH, appears to be the best compromise with respect to location accuracy and computational cost among the set of methods tested in this study. Particularly, if the primary signal can clearly be defined, ESEH is very convenient to use. Despite being slower than ATL and TRL, it is more robust when the signal is noisy, dispersive or fast propagating. This method can be easily applied in 2D environments such as touchscreens or in 3D in the petroleum industry, seismology, or medicine.

See supplementary material S1 for the explanation of the different localization methods used in this study, S2 for detailed information about the numerical simulation, S3 for the details of spatial resolution of EBL, S4 for the optimization of the source energy grid in EBL, S5 for details of the signal to noise ratio comparison, and $\mathrm{S} 6$ for the details of the different array geometries compared.

We would like to thank Alain Steyer and Miloud Talib for the technical support and Maxime Farin for discussions during this work. This project has received funding from the European Union's Seventh Framework Programme for research under grant agreement no 316889, from the REALISE program of the Alsatian research network, from the Universities of Oslo and Strasbourg via a gjesteforsker program and an IDEX Espoirs award. We also thank Jamie I. Farquharson for his help in language to make the article easier to understand.

${ }^{1}$ A. Gershman, V. Turchin, and V. Zverev, Signal Processing, IEEE 43, 2249 (1995).

${ }^{2}$ J. Valin, F. Michaud, J. Rouat, and D. Letourneau, in IROS 2003. Proceedings. IEEE/RSJ, Vol. 2 (2003) pp. 12281233 vol.2.

${ }^{3}$ E. Elnahrawy, X. Li, and R. Martin, in Sensor and Ad Hoc Communications and Networks, IEEE (2004) pp. 406-414.

${ }^{4}$ D. Malioutov, M. Cetin, and A. Willsky, Signal Processing, IEEE 53, 3010 (2005).

${ }^{5}$ Q. Zhu, S. Tannenbaum, and S. H. Kurtzman, Surgical Oncology Clinics of North America 16, 307 (2007), pre and Postoperative Cancer Imaging:Practical and Innovative Approaches.

${ }^{6}$ M. Fink, AIP Conference Proceedings 1650, 13 (2015).

${ }^{7}$ J. Garnier and M. Fink, Wave Motion 53, 80 (2015).

${ }^{8} \mathrm{~K}$. Aki and P. Richards, Quantitative seismology, Vol. 1 (2002).

${ }^{9}$ S. Turkaya, R. Toussaint, F. Eriksen, M. Zecevic, G. Daniel, E. Flekkøy, and K. Måløy, Frontiers in Physics 3 (2015), 10.3389/fphy.2015.00070.

${ }^{10}$ M. J. Niebling, R. Toussaint, E. Flekkøy, and K. Måløy, Revista Cubana de Fisica 29, 1E66 (2012).

${ }^{11}$ O. Lengliné, R. Toussaint, J. Schmittbuhl, J. Elkhoury, J. Ampuero, K. Tallakstad, S. Santucci, and K. Måløy, Physical Review E 84, 036104 (2011).

${ }^{12}$ M. Stojanova, S. Santucci, L. Vanel, and O. Ramos, Physical review letters 112, 115502 (2014).

${ }^{13}$ F. Cornet, J. Helm, H. Poitrenaud, and A. Etchecopar, in Seismicity Associated with Mines, Reservoirs and Fluid Injections (Springer, 1998) pp. 563-583.

${ }^{14}$ K. Evans, F. Cornet, T. Hashida, K. Hayashi, T. Ito, K. Matsuki, and T. Wallroth, Geothermics 28, 455 (1999).

${ }^{15}$ J. Š́lenỳ, Z. Jechumtálová, and C. Dorbath, Pure and Applied Geophysics 171, 2783 (2014).

${ }^{16}$ N. Belayouni, A. Gesret, G. Daniel, and M. Noble, Geophysics 80, WC133 (2015).

${ }^{17}$ G. Carter, Acoustics, Speech, and Signal Processing Newsletter, IEEE 49, 12 (1980).

${ }^{18}$ J. Smith and J. Abel, Acoustics, Speech, and Signal Processing, IEEE 35, 166

${ }^{19} \mathrm{M}$. Brandstein, J. Adcock, and H. Silverman, in Acoustics, Speech, and Signal Processing, Proceedings., IEEE, Vol. 2 (1996) pp. 901-904.

${ }^{20}$ M. Brandstein, J. Adcock, and H. Silverman, Speech and Audio Processing, IEEE 5, 45 (1997).

${ }^{21}$ K. Yao, R. Hudson, C. Reed, D. C., and F. Lorenzelli, Selected Areas in Communications, IEEE 16, 1555 (1998).

${ }^{22} \mathrm{C}$. Reed, R. Hudson, and K. Yao, in Acoustics, Speech, and Signal Processing, Proceedings.,IEEE, Vol. 3 (1999) pp. 1169-1172 vol.3.

${ }^{23} \mathrm{Y} . \mathrm{Hu}$ and D. Li, in Multimedia Signal Processing, IEEE (2002) pp. 371-375.

${ }^{24} \mathrm{X}$. Sheng and Y.-H. $\mathrm{Hu}, \quad$ in Information Processing in Sensor Networks, Lecture Notes in Computer Science, Vol. 2634, edited by F. Zhao and L. Guibas (Springer Berlin Heidelberg, 2003) pp. 285-300.

${ }^{25}$ M. Fink, Ultrasonics, Ferroelectrics, and Frequency Control, IEEE 39, 555 (1992).

${ }^{26}$ C. Hibert, A. Mangeney, G. Grandjean, and N. M. Shapiro, JGR: Earth Surface 116 (2011), 10.1029/2011JF002038, f04032.

${ }^{27}$ M. Farin, A. Mangeney, J. De Rosny, R. Toussaint, J. SainteMarie, and N. Shapiro, Journal of Sound and Vibration 362, 176 (2016).

${ }^{28}$ K. L. Johnson, Contact Mechanics (Cambridge University Press, 1985).

${ }^{29}$ M. Farin, A. Mangeney, R. Toussaint, J. de Rosny, N. Shapiro, T. Dewez, C. Hibert, C. Mathon, O. Sedan, and F. Berger, JGR: Solid Earth 120, 7102 (2015). 